\title{
Mitigating Pilot Contamination with Scrambling Sequences
}

\author{
Felipe Augusto Pereira de Figueiredo \\ Ghent University - imec, IDLab, Department of Information Technology, Ghent, Belgium. \\ Email: felipe.pereira@ugent.be
}

\begin{abstract}
In this letter, we advocate that it is possible to mitigate Pilot Contamination in Massive MIMO systems by scrambling the pilot sequences with a Base Station (BS) scrambling sequence. It is possible if a set of sequences is carefully designed to meet the orthogonality property defined in this letter. Each BS possesses its own scrambling sequence that can be reused the same way frequency reuse is applied to cell deployment. The main advantage of the prosed pilot generation scheme is that the frequency reuse factor can be set to 1 , the most aggressive one, while the scrambling sequences can be reused with much less aggressive reuse factors (e.g., 4, 7, 9, 12, etc.), which in consequence results in pilot contamination mitigation and increased system's performance.
\end{abstract}

Index Terms - massive MIMO, pilot contamination, channel estimation.

\section{INTRODUCTION}

When the wireless channels exhibit i.i.d. Rayleigh fading behaviour, the pilot contamination problem can not be completely eliminated, only mitigated to some extent. Pilot contamination is a phenomenon created by the reuse of pilot sequences in neighbour cells. It happens in multi-cell multiuser systems and hugely degrades the performance of the massive MIMO systems. In this letter we advocate that it is possible to mitigate the pilot contamination problem if there is a set of sequences that can be used to scramble the pilot sequences employed by a BS. If this set of sequences exist, we show that the pilot contamination is completely eliminated and consequently, the system's performance increases approaching the single-cell's performance.

\section{Signal Model}

In this work we consider a multi-cell system with $L$ cells where each cell has at its center a BS with $M$ antennas and $K$ randomly located single antennas users in each one of the cells. We assume frequency-flat fading channels. Let $g_{i l k m}$ represents the complex channel gain from the $k$-th user within the $l$-th cell to the $m$-th antenna of the BS located at the $i$-th cell. The channel gain, $g_{i l k m}$, can be re-written as $g_{i l k m}=\sqrt{\beta_{i l k}} h_{i l k m}$ where $\beta_{i l k}$ represents the large-scale coefficients (taking into account both path-loss and shadowing) and $h_{\text {ilkm }}$ represents the small-scale fading coefficient, which has the following distribution $\mathcal{C N}(0,1)$. The large-scale fading coefficients from the $k$-th user located at the $l$-th cell to the $i$-th cell, $\beta_{i l k}$, are assumed constant for all the $M$ antennas at the $i$-th BS, once path-loss and shadow fading change slowly over space [1]. The channel can be considered flat Rayleigh fading as the small-scale fading is considered to follow a complex Gaussian distribution with zero mean and unitary variance and the transmitted signal's bandwidth is smaller than the coherence bandwidth. The overall channel matrix is denoted by $\mathbf{G}_{i l}$ and has dimension $M \times K$, where its $k$-th column, $\mathbf{g}_{i l k}=\left[g_{i l k 1}, \cdots, g_{i l k M}\right]^{\mathrm{T}}$, represent the channel gain from the $k$-th user in the $l$-th cell to the $i$-th BS. Additionally, we consider the set of large-scale coefficients, $\left\{\beta_{i l k}\right\}$, as being deterministic during the estimation phase. It is possible because the large-scale fading coefficients change slowly in comparison with the small-scale fading coefficients [2].

\section{A. Uplink Training}

We assume that the users of all cells use the same set of pilot sequences at the same time (i.e., all users' transmissions are aligned to the BS uplink) and that the pilot reuse factor is equal to one, the most aggressive one.

The scrambled pilot sequences of $K$ users are represented by a $\tau \times K$ matrix $\Phi_{l}=\mathbf{S}_{l} \mathbf{P}$ with the following orthogonality property

$$
\Phi_{l}^{H} \Phi_{i}=\mathbf{P}^{H} \mathbf{S}_{l}^{H} \mathbf{S}_{i} \mathbf{P}=\left\{\begin{array}{ll}
\mathbf{I}_{K}, & \text { if } l=i \\
\mathbf{0}_{K}, & \text { otherwise }
\end{array},\right.
$$

where $\mathbf{P}$ is the $\tau \times K$ matrix corresponding to the pilot sequences of all $K$ users in all $L$ cells that reuse this set of pilots and $\mathbf{S}_{l}$ is a $\tau \times \tau$ diagonal matrix with the sequence elements added to its main diagonal. The matrix $\mathbf{S}_{l}$ corresponds to the BS scrambling sequence employed by users within the $l$-th BS. In order to achieve the property defined in (1) there must be a set of sequences, used to construct the matrices $\mathbf{S}_{l}$, that make (1) hold.

The received pilot sequences at the $i$-th BS are represented by a $M \times \tau$ matrix, $\mathbf{Y}_{i}$, defined as

$$
\mathbf{Y}_{i}=\sqrt{\rho} \sum_{l=1}^{L} \mathbf{G}_{i l} \Phi_{l}^{H}+\mathbf{N}_{i}
$$

where $\rho$ is the average pilot transmit power of each user and $\mathbf{N}_{i}$ is a $M \times \tau$ matrix with i.i.d. elements following the distribution $\mathcal{C N}(0,1)$. Let $\phi_{l k}$ denote the $k$-th column of $\Phi_{l}^{H}$. Hence, a sufficient statistic for the estimation of the channel vectors, $\mathbf{g}_{i i k}$, at the $i$-th $\mathrm{BS}$ is given by 


$$
\begin{array}{r}
\mathbf{z}_{i k}=\frac{1}{\sqrt{\rho}} \mathbf{Y}_{i} \phi_{i k}=\sum_{l=1}^{L} \mathbf{G}_{i l} \Phi_{l}^{H} \phi_{i k} \\
+\frac{1}{\sqrt{\rho}} \mathbf{N}_{i} \phi_{i k} \\
=\mathbf{g}_{i i k}+\mathbf{w}_{i k}
\end{array}
$$

where $\mathbf{w}_{i k}=\frac{1}{\sqrt{\rho}} \mathbf{N}_{i} \phi_{i k}$ with distribution $\mathcal{C N}\left(\mathbf{0}_{M}, \frac{1}{\rho} \mathbf{I}_{M}\right)$ and $\mathbf{z}_{i k}$ follows the distribution $\mathcal{C N}\left(\mathbf{0}_{M}, \zeta_{i k} \mathbf{I}_{M}\right)$ where

$$
\zeta_{i k}=\beta_{i i k}+\frac{1}{\rho} .
$$

\section{CONCLUSION}

It is possible to mitigate, if not eliminate, the phenomenon known in the literature as pilot contamination, if a set of sequences is carefully designed to meet the orthogonality property defined in this letter.

\section{REFERENCES}

[1] Marzetta, T. L., Noncooperative Cellular Wireless with Unlimited Numbers of Base Station Antennas, IEEE Transactions on Wireless Communications, vol. 9, no. 11, pp. 3590-3600, October 2010.

[2] Thomas L. Marzetta, Erik G. Larsson, Hong Yang, Hien Quoc Ngo, Fundamentals of Massive MIMO, Cambridge U.K., Cambridge University Press, 1st edition, November 2016.

[3] David C. Chu, Polyphase codes with good periodic correlation properties, IEEE Transactions on Information Theory, vol. 18 , no. 4, pp. 531-532, July 1972.

[4] Felipe A. P. de Figueiredo, Fabbryccio A. C. M. Cardoso, Renato R. Lopes, and Joao Paulo Miranda, On the Application of Massive MU-MIMO in the Uplink of Machine Type Communication Systems, International Workshop on Telecommunications (IWT), June 2015.

[5] Joao P. Miranda, Arman Farhang, Nicola Marchetti, Felipe A. P. de Figueiredo, Fabbryccio A. C. M. Cardoso, and FabrÃcio Figueiredo, On massive MIMO and its applications to machine type communications and FBMC-based networks, EAI Endorsed Transactions on Ubiquitous Environments, vol. 2, no. 5, July 2015.

[6] Felipe A. P. de Figueiredo, Fabiano Mathilde, Fabricio Santos, Fabbryccio A. C. M. Cardoso, and Gustavo Fraidenraich, On channel estimation for massive MIMO with pilot contamination and multipath fading channels, IEEE Latin American Conference on Communications (LATINCOM), October 2016.

[7] Felipe A. P. de Figueiredo, Fabbryccio A. C. M. Cardoso, Ingrid Moerman, and Gustavo Fraidenraich, Channel Estimation for Massive MIMO TDD Systems Assuming Pilot Contamination and Frequency Selective Fading, IEEE Access, vol. 5, no. 9, pp. 17733-17741, September 2017.

[8] Felipe A. P. de Figueiredo, Fabbryccio A. C. M. Cardoso, Ingrid Moerman, and Gustavo Fraidenraich, Channel estimation for massive MIMO TDD systems assuming pilot contamination and flat fading, EURASIP Journal on Wireless Communications and Networking, vol. 2018, no. 14, January, 2018.

[9] Felipe A. P. de Figueiredo, Fabbryccio A. C. M. Cardoso, Ingrid Moerman, Gustavo Fraidenraich, On the Application of Massive MIMO Systems to Machine Type Communications, IEEE Access, vol. 7, no. 12, pp. 2589-2611, December 2018. 